\title{
BINARY STARS AND PLANETARY NEBULAE
}

\author{
Icko Iben, Jr.* \\ Dept. of Astronomy, University of Illinois, $1011 \mathrm{~W}$. \\ Springfield, Urbana, IL 61801 \\ Alexander V. Tutukov** \\ Astronomical Council, USSR Academy of Sciences
}

\begin{abstract}
A non-negligible ( 15-208) fraction of planetary nebulae is expected to be formed in close binaries in which one component fills its Roche lobe after the exhaustion of hydrogen or helium at its center. The nebula is ejected as a consequence of a frictional interaction between the stellar cores and a common envelope; the ionizing component of the central binary star may be a relatively high luminosity contracting star with a degenerate $C O$ core, burning hydrogen or helium in a shell, or it may be a lower luminosity shell hydrogen-burning star with a degenerate helium core or a core helium-burning star. Even more exotic ionizing central stars are possible. Once the initial primary has become a white dwarf or neutron star, the secondary, after exhausting central hydrogen, will also fill its Roche lobe and eject a nebular shell in a common envelope event. The secondary becomes the ionizing star in a tight orbit with its compact companion. In all, there are roughly twenty different possibilities for the make-up of binary central stars, with the ionizing component being a post asymptotic giant branch star with a hydrogen- or heliumburning shell, a CO dwarf, a core helium-burning star, a shell helium-burning star with a degenerate CO core, a shell hydrogenburning star with a degenerate helium core, or a helium degenerate dwarf, while its companion is a main sequence star, a CO degenerate dwarf, a helium star, a helium degenerate dwarf, or a neutron star. We estimate the occurrence frequency of several of these types and comment on the prior evolutionary history of 4 observed binary central stars.
\end{abstract}

\footnotetext{
"Supported in part by the United States NSF Grant AST 84-13771.

** Supported in part by the Instituto de Astronomia, University of Mexico, and by the USSR Academy of Sciences.
} 


\section{INTRODUCTION}

It is commonly assumed that most planetary nebulae (PNe) originate from single stars which eject a shell of matter after they have exhausted helium in their cores and have become asymptotic giant branch (AGB) stars. The nature of the ejection mechanism has yet to be satisfactorily identified, but it may involve a secular instability in the envelope itself (Wood 1974; Tuchman, Sack, and Barkat 1979) and/or the development of a wind driven by pulsational energy and by radiation pressure on grains (Wood 1981, Willson 1986, Draine 1981, Fadeev 1986). A low mass representative reaches the thermally pulsing (TP) AGB phase and develops a chemically evolved surface composition and may pass through a Mira phase before ejecting most of its hydrogen-rich envelope. In the case of more massive progenitors, the ejection event may be evident in the OH/IR phenomenon. Much of this evolution is described in Kwok and Pottasch (1987).

The conventional picture is, of course, an oversimplification. In a significant fraction of all cases, membership in a binary may play an important if not crucial role either in ejecting matter or in influencing the morphology and chemistry of the extended nebula, or both. Theory suggests that many close binaries (initial orbital separation less than 1500R ) should experience one or more common envelope events during which they eject from the system most of the mass of one of the stars, which thereafter evolves either directly into a luminous, hot $(>100,000 \mathrm{~K}$ ) shell nuclear burning star (which can cause the ejected material to fluoresce) and then into a white dwarf, or first into a less luminous and cooler helium star (which also can, if hot enough, cause nebular fluorescence).

The characteristics of the exciting central star and of its companion can conspire against the serendipitous detection of duplicity. The central star is, as a rule, extremely bright and lines in its spectrum are rather broad, making it difficult with conventional techniques to detect a relatively dim main sequence or degenerate dwarf companion. Only the shortest period systems with a favorable orientation of orbital plane can be detected as eclipsing binaries or as variable stars in which the intrinsically dim component is illuminated by its bolometrically brighter companion.

To estimate the probabilities of different binary evolutionary scenarios we need to know the initial distribution of newly forming binary stars with respect to semimajor axis $A_{0}$ and primary mass $M_{1}$. In first approximation, this is given by (Popova et al. 1982, Iben and Tutukov 1984a, 1985)

$$
d^{2} \mathrm{~N} \sim 0.1 \operatorname{dlog} \mathrm{A}_{0} \mathrm{dM}_{1} / \mathrm{M}_{1}^{2.5} \mathrm{yr}^{-1},
$$

where $A_{0}$ and $M_{1}$ are in solar units (which we shall use hereinafter, unless otherwise specified). This relationship is valid for $1<\log A_{0}<6$ and $1<M_{1}<100$. The numerical value of the coef- 
ficient in equation ( 1 ) is uncertain by at least a factor of two and the uncertain dependence on mass ratio has been suppressed. From this equation it follows that almost all planetary nebula nuclei (PNNi) may be in binaries, with the separation of components on occasion being of the order of the nebular size itself. However, only about 15-20\% of all initial binaries will be close enough $(A<1500)$ that the primary will, after exhausting central hydrogen, fill its Roche lobe before it can reach the thermally pulsing AGB phase and eject a PN shell of its own accord. An even larger percentage may be far enough apart to avoid mass loss by Roche-lobe overflow, but close enough to exercise some shaping influence on the nebula emitted by one of the stars.

Curiously enough, approximately 158 of all central stars that have been examined carefully for duplicity are binaries with periods less than 1 day (Bond 1987, Ritter 1987, and references therein) and Bond (1987, this conference) infers that a much larger percentage of planetaries contains close binary central stars with periods larger than 1 day, with perhaps all PNe containing close binary central stars. We suspect that the apparent high frequency of very short period binary stars may be a consequence of selection.

Most early computations of close binary evolution were carried out in the conservative approximation (total mass = constant, total angular momentum $=$ constant). Now it is known that, in binaries with initial mass ratio $q_{0}\left(=M_{1} / M_{2}\right)$ exceeding $\sim 2$ when the donor has a radiative envelope and $\sim 0.6$ when the donor has a convective envelope, Roche-lobe filling leads to common envelope formation ([Ostriker and] Paczynski 1976, Meyer and MeyerHofmeister 1979, Tutukov et al. 1982). Matter entering the common envelope is driven from the system because of friction between the central binary and the common envelope. Most of the matter originally in the hydrogen-rich surface layers of the primary is in this way lost from the system. Mass loss ceases when the compact remnant of the primary shrinks within its Roche lobe.

After the loss of the common envelope, the system consists of the essentially unaltered secondary and a compact remnant, the two stars being much closer together than the original pair. If it becomes hot enough quickly enough, the remnant can ionize the ejected common envelope material, and the system can be recognized as a PN. In the further course of evolution, as many as three additional major common envelope events may occur, each event leading to a decrease in orbital separation. After each ejection a PN phase may occur.

The reduction in semimajor axis during the common envelope stage can be estimated from the expression (Tutukov and Yungelson 1979)

$$
M_{1}^{2} / A_{0} \sim \alpha M_{2} M_{1 R} / A_{f}
$$

where $A_{0}$ is the semimajor axis before the common envelope event, $A_{f}$ is the final semimajor axis, $M_{1}$ is the initial primary mass, 
$M_{1 R}$ is the mass of its remnant, $M_{2}$ is the mass of the companion, and $\alpha$ is the "efficiency". Equation (2) permits us to estimate the semimajor axis after each successive common envelope event which the system experiences during the evolution of its components and equations (1) and (2) together may be used to estimate birth rates of different types of binaries at centers of planetary nebulae.

Equation (2) follows from the fact that energy is required to drive matter against gravity from the "surface" of the donor, through the common envelope, and endow this matter with sufficient kinetic energy to escape from the system. A measure of the energy required to eject all of the matter $M_{10 s t}$ lost by the primary may be written as $\delta \mathrm{E}_{\text {eject }} \sim \mathrm{GM}_{10 \text { st }}\left\langle\mathrm{M}_{\mathrm{stars}}>/ \mathrm{A}_{0}\right.$, and a measure of the change in orbital binding energy may be written as $\delta \mathrm{E}_{\mathrm{b} \text { ind }}$ $\mathrm{GM}_{1 \mathrm{R}} \mathrm{M}_{2} / 2 \mathrm{~A}_{\mathrm{f}}-\mathrm{GM}_{1} \mathrm{M}_{2} / 2 \mathrm{~A}_{0}$. Here $\left\langle\mathrm{M}_{\mathrm{stars}}\right\rangle$ is the mean mass of the stellar system from which matter is escaping, and we have assumed that the secondary has not gained any mass. We may write $M_{10 s t}=$ $M_{1}-M_{1 R}$ and $\left\langle M_{\text {stars }}>\sim\left(M_{1}+M_{1 R}\right) / 2+M_{2}\right.$. If $M_{1}$ is large compared with both $M_{1 R}$ and $M_{2}$, setting $\delta E_{\text {eject }} \sim \delta E_{b_{i n d}}$ gives $M_{1}{ }^{2} / A_{0}$ $\sim M_{2} M_{1 R} / A_{f}$. If we assume that $M_{1} \sim M_{2}$, but still retain the assumption of small $M_{1 R} / M_{1}$, the same argument gives $M_{1}^{2} / A_{0}-(1 / 4)$ $M_{2} M_{1 R} / A_{f}$. Thus, $\alpha$ in equation (2) is a parameter of order $0.2-1$, subject, of course, to the validity of the assumption that orbital energy is efficiently used up in driving off matter in the common envelope.

Several attempts have been made to model a common envelope event using two dimensional hydrodynamics (Bodenheimer and Taam 1984, Bond and Livio 1987, Hachisu 1987), and these studies suggest that a large fraction of the orbital energy goes into escaping radiation and into producing large terminal velocities, making $\alpha$ considerably smaller than unity. It must be cautioned, however, that the problem is three dimensional and important physical processes, such as the transport of angular momentum by turbulent viscosity, have been left out of the calculations. Further, with $\alpha$ as small as, say, 0.1 , it becomes difficult to understand the formation of cataclysmic variables.

In the following sections we will (II) explore the various possibilities for PN formation in close binaries and estimate the occurrence frequencies for several types; (III) attempt to divine the nature of the progenitors of several observed PNe with binary central stars; and (IV) compare and contrast the morphology, chemical composition, brightness, and lifetimes of PNe formed by single stars with those formed by close binaries in common envelope events.

2. TYPES AND FORMATION FREQUENCIES OF PLANETARY NEBULAE IN CLOSE BINARIES

The various possibilities for $\mathrm{PN}$ formation may be discussed best with reference to Fig. 1, where we summarize critical orbital 


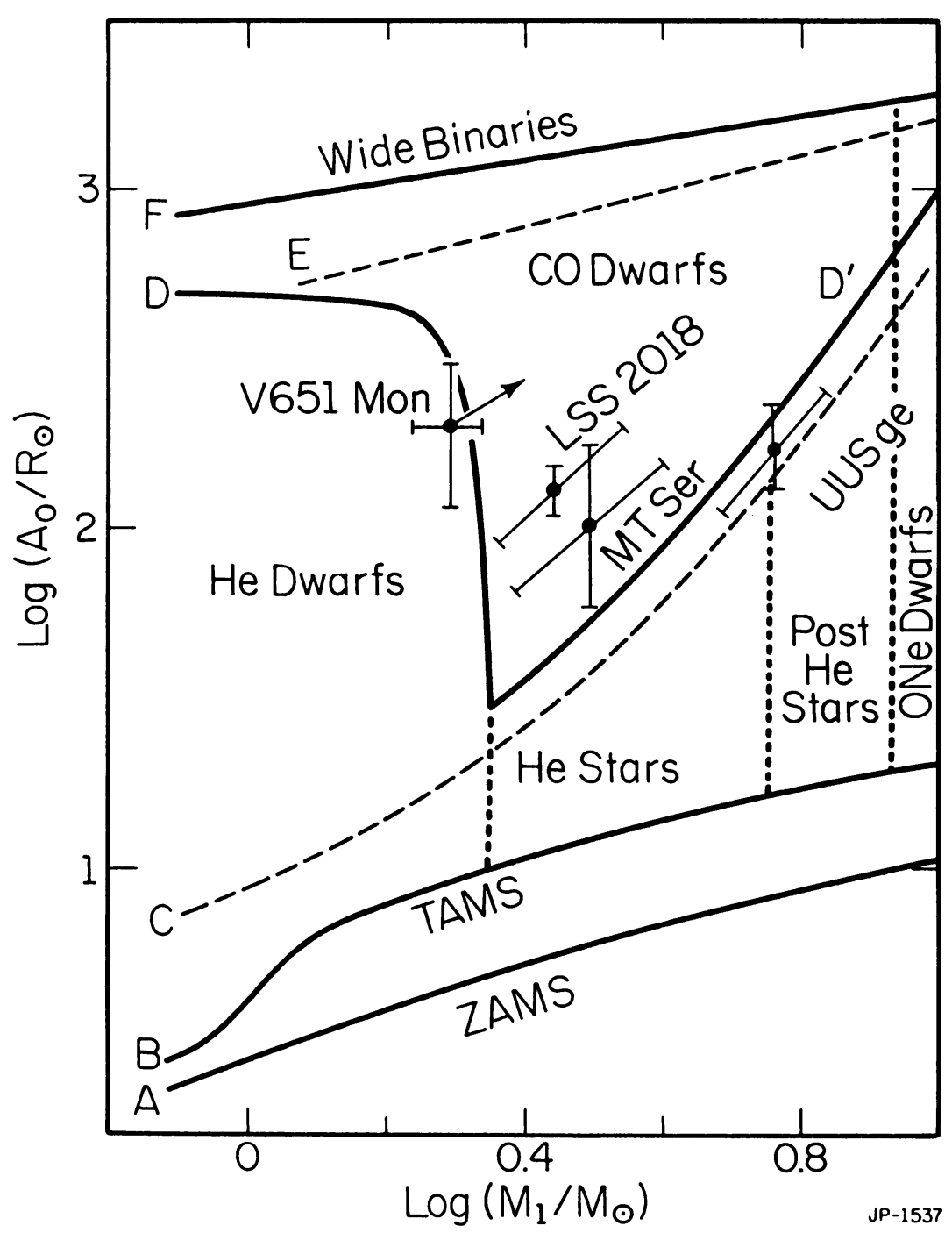

Figure 1. Critical borders influencing the nature of the mass exchange process and the nature of the remnants in close binaries. $A_{0}=$ orbital separation and $M_{1}=$ primary mass. $F$ is the maximum separation for interaction, $E$ is the start of the TP-AGB phase, D is where the primary ignites helium in its core, $C$ is the border between systems in which the primary has a radiative envelope and those in which it has a deep convective envelope. Curves A and B are main sequence boundaries. The dotted lines are separate systems whose components produce different remnants. 
separations as a function of the initial primary mass. Curve A gives the minimum separation $A_{0}$ that allows a zero age main sequence primary to fit within its Roche lobe, and curve $B$ gives the separation allowing a terminal age main sequence primary to fit within its Roche lobe. For systems with $A_{0}$ below curve $C$, the primary has a large radiative envelope, and for those with $A_{0}$ above curve $C$, the primary has a deep convective envelope. For systems with $A_{0}$ larger than on curve $D$, the primary ignites helium in its core (which is degenerate if $M_{1}<2.3$ and non degenerate otherwise) and cannot fill its Roche lobe until after it has exhausted central helium and become an AGB star. For systems with $A_{0}$ between curves $D$ and $E$, the primary fills its Roche lobe after it has developed a degenerate CO core, but before it has begun to experience thermal pulses. For systems with $A_{0}$ between curves $E$ and $F$, the primary fills its Roche lobe while in the TP-AGB phase. Finally, in systems with $A_{0}$ larger than on curve $F$, the primary ejects its hydrogen-rich envelope of its own accord, largely uninfluenced by being in a binary.

The locations of the critical curves are to be considered only as rough qualitative guides. Not only has the dependence of critical separations on the mass ratio prior to each common envelope event been suppressed, but the critical separations are functions of the composition and input physics. More details are described in Iben and Tutukov $(1985,1987)$. We will concentrate first on what happens as a consequence of the initial Roche-lobe overflow event.

In systems bounded by. curves $B$ and $C$, if $q_{0} \sim 1$, the thermal time scales of the radiative envelopes of both components are comparable when the primary first fills its Roche lobe, and it is probable that mass transfer will not lead to the establishment of a common envelope. The net result may be the transfer of much of the hydrogen-rich envelope of the primary to the secondary and an increase in the orbital separation. Evolution of this sort explains the properties of low mass Algols such as RY Gem ( $M_{t o t}$ 2.6) and $X Y$ Pup $\left(M_{t \circ t} \sim 2.3\right)$. In these systems, the initial primary was probably of mass less than 2.3 and did not fill its Roche lobe until after it had developed an electron-degenerate helium core. More massive Algols such as $U$ Sge $\left(M_{t o t} \sim 7.6\right)$ and RS Vul ( $\left.M_{t \circ t} \sim 5.9\right)$, which derive from systems with $M_{1}>2.3$, have probably experienced conservative mass transfer as well; even though the helium core of the primary was not degenerate at the start of mass transfer, it ultimately became degenerate in the course of mass loss. This must be so, since, in both instances, the primary has evolved into a subgiant. In Algols, mass transfer continues at a rate controlled by angular momentum loss due to a magnetic stellar wind (Verbunt and Zwaan 1981). When the mass in its hydrogen-rich envelope has been reduced sufficiently, the primary will contract within its Roche lobe and, when shell hydrogen burning in it ceases, it will evolve into a helium white dwarf. The more massive secondary continues for a while to burn hydrogen as a main sequence star. 
In neither case does a PN phase follow the inital massreversing mass-transfer phase. Not only is there very little mass ejected to be ionized, but the primary, which contains a degenerate helium core, does not become hot enough to ionize what little mass is ejected until after it has evolved for millions of years at low surface temperatures burning hydrogen in a shell.

A better chance for PN formation is presented by systems bounded by curves $C$ and $D$ and by $2.3<M_{1}<10$ in Fig. 1 . In such systems, the primary, with a non degenerate helium core, will have developed a deep convective envelope before filling its Roche lobe. After ejecting most of its hydrogen-rich envelope through its Roche lobe, it will evolve for a time as a compact core helium-burning star. If $q_{0}$ is large enough so that envelope shrinkage forces the remnant of the primary to have a surface temperature $>30,000 \mathrm{~K}$ as it shrinks within its Roche lobe, the ejected hydrogen-rich material will become ionized and a PN will result. If $M_{1}<5$, the primary remnant, of mass $<0.75$, will remain within its Roche lobe as it exhausts helium at its center and evolves eventually into a CO dwarf. If $M_{1 R}<0.4$, both hydrogen and helium shell flashes occur (Iben and Tutukov 1985, Iben et al. 1986) and there is the possibility of the ejection of a nebula of mass $\sim 10^{-4} \mathrm{M} \odot$.

If $M_{1}$ is between $5^{\odot}$ and 10 , the remnant of the primary will, after it has exhausted central helium, swell again to fill its Roche lobe. In a second mass loss event, a common envelope is again formed and mass loss from the system continues until the mass of the primary remnant is reduced to $\sim$ 0.75-1.1. The ejected material, of mass between $\sim 0$ and 1.4 is essentially pure helium (with a small admixture of ${ }^{14} \mathrm{~N}$ ). The remnant burns helium in a shell on a time scale of $\sim 10^{4} \mathrm{yr}$ at high luminosity (few $\times 10^{4} \mathrm{~L}_{\odot}$ ) and at temperatures large enough to cause the helium-rich nebula to fluoresce (Iben and Tutukov 1985). Similar behavior is expected from systems with $A_{0}$ less than along curve $C$ in Fig. 1 , if $q_{0}$ is large enough.

Other possible sources of observable PNe are systems bounded by curves $C$ and $D$ and by $M_{1}<2.3$ in Fig. 1 . The primary star has both an electron degenerate helium core and a deep convective envelope. Roche-lobe overfilling will therefore lead to a common envelope and loss from the system of most of the hydrogen-rich envelope of the primary. The ejected envelope can potentially be ionized by the shell hydrogen-burning remnant. From published models, however, one might infer that the remnant spends so much time contracting at temperatures less than $30000 \mathrm{~K}$, that the ejected nebular material will be dispersed before the remnant becomes hot enough to excite the nebula into fluorescence (Iben and Tutukov 1986a). Configurations such as these have been dubbed "lazy" PNe by Renzini $(1979,1983)$. Since the remnant may eventually achieve temperatures far in excess of $30,000 \mathrm{~K}$ on a long time scale and at a relatively large luminosity, we have the interesting situation of a very hot and bolometrically bright star at the center of an invisible, or at least very dim, nebula. 
The situation may not be as bleak as this. The published models do not explicitly take into account the shrinkage of the Roche lobe about the primary and the possible dynamical motions induced in the envelope of the mass-losing primary. When the primary first fills its Roche lobe, its luminosity and radius are related to the mass $M_{H e}$ of its degenerate helium core by

$$
\mathrm{L}_{1} \sim 10^{5 \cdot 5} \mathrm{M}_{\mathrm{He}}{ }^{6 \cdot 6}, \mathrm{R}_{1} \sim 10^{3} \cdot{ }^{5} \mathrm{M}_{\mathrm{He}}{ }^{4} \text {. }
$$

The luminosity of the remnant as it emerges from the common envelope event is approximately $L_{P N N} \sim L_{1}$, its mass is $M_{P N N} \sim M_{H e}$, and its radius $R_{P N N}$ is related to its surface temperature $T_{P N N}$ by

$$
\mathrm{R}_{\mathrm{PNN}} \sim 10^{1.32} \mathrm{M}_{\mathrm{PNN}}{ }^{3.3}\left(30000 \mathrm{~K} / \mathrm{T}_{\mathrm{PNN}}\right)^{2} .
$$

This tells us that, if the radius of the Roche lobe of the remnant as it emerges from the common envelope phase is smaller than, say, 1.5 (if $M_{P N N} \sim 0.45$ ), or 0.4 (if $M_{P N N} \sim 0.3$ ), a $P N$ phase with $L_{P N}$ $\sim \mathrm{L}_{1}$ is ensured.

Consider a system with $M_{1}=1.5, M_{2}=0.5$, and $A_{0}=283$. The Roche-lobe radius of the primary is given approximately by

$$
R_{1 L} \sim 0.52\left(M_{1} / M_{t \circ t}\right)^{0.44} A_{0}
$$

where $M_{\text {tot }}=M_{1}+M_{2}$. Combining equations (3) and (5), we have that the primary fills its Roche lobe when its core mass reaches 0.45 . Equations (3) and (2) tell us that, following an assumed common envelope phase, $A_{f}-15.9 \alpha$ and the radius of the Roche lobe of the remnant is $R_{1 \mathrm{~L}, \mathrm{f}} \sim 6 \alpha$. Since nebular excitation requires that $\mathrm{T}_{\mathrm{PNN}}>30000 \mathrm{~K}$, we conclude that a PN phase will not immediately follow the common envelope event, if indeed it occurs at all, unless $\alpha<0.25$. Similarly, choosing $M_{1}=1.5, M_{2}=0.5$, and $A_{0}=$ 56 , we find $M_{1 R} \sim M_{H e}=0.3, A_{f}-2.1 \alpha$, and $R_{1 L, f} \sim 0.7 \alpha$ and conclude that a PN phase is ensured only if $\alpha<0.6$.

Actually, as the common envelope event nears its close, the radius of the primary remnant may (due to dynamical effects alluded to earlier) be inflated considerably beyond that of a formal quasistatic model of the same luminosity and mass and, following Roche-lobe detachment, the radius may shrink on a time scale much smaller than given by the quasistatic model (for an elaboration of this theme, see our discusion in III of UU Sge). Hence, the upper limits on $\alpha$ which we have estimated are only suggestive and serve primarily to indicate that (1) whether or not a PN phase follows the common envelope phase is very sensitive to $\alpha$, and (2) all other things being equal, the smaller the mass of the remnant relative to the mass of its progenitor, the more likely is the formation of a PN immediately following the first common envelope event in a low mass system.

This second statement is true only up to a point. There is a maximum surface temperature $T_{\max }$ which a remnant can achieve and this maximum is smaller, the smaller the mass of the remnant. If 
the remnant eventually evolves quasistatically, burning hydrogen in a shell, the maximum may be estimated quantitatively:

$$
\mathrm{T}_{\max } \sim 10^{5 \cdot 5} \mathrm{M}_{\mathrm{PNN}}{ }^{1.8} .
$$

To obtain this estimate, we have normalized to the $0.3 \mathrm{M}_{\odot}$ model of Iben and Tutukov (1986a), assuming that the luminosity at $\mathrm{T}_{\mathrm{max}}$ has the same mass dependence as does $L_{1}$ in equation (3) and that the radius at $T_{m a x}$ has the same mass dependence as the radius of a low mass white dwarf, namely $R \propto M_{P N N}{ }^{-1 / 3}$. For the $0.3 M_{\odot}$ model, the luminosity at $\mathrm{T}_{\mathrm{max}}$ is approximately $\mathrm{L}_{1} / 2.6$ and the radius is approximately $5.7 \mathrm{R}_{\mathrm{WD}}$, where $\mathrm{R}_{\mathrm{WD}} \cong 10^{-1.9} \mathrm{M}_{\mathrm{PNN}}$ is the final radius of the model after it has become a white dwarf. We conclude that the mass of the remnant must be larger than $0.27 \mathrm{M}_{\odot}$ if the ejected nebula is to become ionized.

In systems bounded by the curves $E$ and $F$ in Fig. 1, the primary will have developed a degenerate CO core before it fills its Roche lobe and will also have a very deep convective envelope. One therefore expects a common envelope to be formed when Rochelobe overflow occurs. Since it is in the thermally pulsing stage, the primary may have developed chemical peculiarities at its surface. We note that the "phase space" for the formation of such systems (the area between curves $\mathrm{E}$ and $\mathrm{F}$ is quite small, so they arise infrequently.

Consider next what happens after the initial primary has evolved into a degenerate dwarf (or neutron star). We refer again to Fig. 1 as a qualitative guide, replacing $A_{0}$ by $A_{f}$ and $M_{1}$ by $M_{2}$, and remember that, if $A_{0}$ is between curves $C$ and $F$, then $A_{f}$ is much smaller than $A_{0}$ as a result of common envelope action. When using equation (2), we must replace $M_{1 R}$ by $M_{2 R}, M_{2}$ by $M_{1 R}$, and $M_{1}$ by $M_{2}$. In all cases, $A_{f}$ is expected to be less than given by curve $D$, and in many instances less than given by curve $C$ (unless the initial mass-transfer was conservative). This time, however, the potential accretor is a compact white dwarf (or sometimes a helium star or a neutron star) and accretion at a rate larger than the Eddington accretion limit $\left(-10^{-6} \mathrm{M}_{\odot} \mathrm{yr}^{-1}\right.$ for a $1 \mathrm{M}$ white dwarf) will always cause the accreted layer to swell to fill the compact object's Roche lobe, so that a common envelope is formed. Even if the donor has a radiative envelope when it fills its Roche lobe, it is expected that mass transfer rates will in general exceed the Eddington limit. Both because a common envelope is formed and because the mass ratio of donor to accretor is frequently large, considerable orbital shrinkage will occur and the remnant of the donor should quickly become hot enough to ionize the ejected material.

If $A_{f}$ is between curves $B$ and $C$ and $M_{2}<2.3$ in Fig. 1 , the secondary will become a shell hydrogen-burning star with a degenerate helium core and ultimately evolve into a degenerate helium dwarf. If $M_{2}-2.3-5$, the secondary will become a compact core helium burning star before evolving into a $\mathrm{CO}$ degenerate dwarf. If $M_{2} \sim 5-10$, the secondary will swell to fill its Roche 
lobe and, in a common envelope event, will eject a helium envelope which it will ionize as a shell helium burning star with a degenerate $\mathrm{CO}$ core.

To summarize, our evolutionary scenarios suggest that the ionizing star after the first mass exchange may be (1) shell hydrogen-burning star with a degenerate helium core, (2) a helium star, (3) a post helium star object with a helium-burning shell and a degenerate CO core, (4) a post AGB star with a hydrogen or helium burning shell, and (5) a helium or CO degenerate dwarf. Possibilities (1)-(3) can occur only in binaries. In case (3), the matter of the $P N$ is pure helium. Possibilities (4) and (5) differ from single-star PNe in the manner of ejection of nebular material. After the second mass loss event, the ionizing star may be one of possibilities (1), (2), (3), or (5), while its companion may be (1) a CO dwarf, (2) a helium dwarf, (3) a helium star, or (4) a neutron star. There are also cases in which the central stars merge in the process of ejecting a common envelope, but we decline to explore this possibility further here.

The formation frequency of the most likely variants can be estimated with the help of equation (1) and Fig. 1. Equation (1) implies that almost all stars are binaries and that the frequency of PN formation around components of all binaries (most of which begin and remain wide) is $\sim 0.56 \mathrm{yr}^{-1}$. We estimate a formation rate of $0.065 \mathrm{yr}^{-1}$ for $\mathrm{PNe}$ with central stars consisting of a shell hydrogen-burning star with a degenerate CO core (or its CO white dwarf descendant) and a main sequence star and a formation rate of $\sim 0.007 \mathrm{yr}^{-1}$ for PNe consisting of a post core helium-burning star with a degenerate $C O$ core (or its $C O$ white dwarf descendant) and a main sequence star. About ten percent of these two types of systems evolve eventually into cataclysmics. The second type forms a helium-rich nebula. In about 18 of all cases the central star may be a core helium-burning star, but this may be a gross underestimate since, if $q_{0}$ is large, common envelope events occur even if $A_{0}$ is below curve $C$.

About one tenth of all post common envelope systems consisting of a hot compact star and a main sequence star will evolve by angular momentum loss into cataclysmic variables. Two conditions must be satisfied for this transformation to occur. First, $q_{0}<1.2$ if $M_{2}>0.8$ and $q_{0}<0.6$ if $M_{2}<0.8$. Second, there are restrictions on $A_{f}$. If $M_{2}<0.3$, the secondary is completely convective and the magnetic stellar wind (MSW) is weak. So, only gravitational wave radiation (GWR) can drive the components closer together; only if the period of the system after emerging from the common envelope satisfies $P<5^{\mathrm{h}} \mathrm{M}_{2}{ }^{3 / 8}$, will the system become a cataclysmic variable in less than $10^{10} \mathrm{yr}$. If 0.3 $<M_{2}<1.5$, the MSW can drive the components together in less than $10^{10}$ yr if $\mathrm{P}<32^{\mathrm{h}} \mathrm{M}_{2}{ }^{1.2}$. These limits are discussed in more detail by Iben and Tutukov (1984b), for example.

After the second common envelope event, the most probable systems will be a shell hydrogen-burning star with a co core in orbit with a CO degenerate dwarf deriving from systems with $2.3<$ 
$M_{1}<4,2<\log A_{0}<3$, at a formation frequency of $0.015 \mathrm{yr}^{-1}$, and systems of $\mathrm{CO}$ and He degenerate dwarfs (one of which may have a hydrogen-burning shel1) deriving from systems with $2.3<M_{1}<4$, $1<\log A_{0}<1.5$, at a frequency of $\sim 0.005 \mathrm{yr}^{-1}$. The total frequency of close binaries as PNNi is thus estimated to be about $0.10 \mathrm{yr}^{-1}$ or about 208 of the total frequency of PN formation.

Systems with $A_{0}<500$ and $M_{1}<2.3$ form double degenerate helium dwarfs (Iben and Tutukov 1986b). Their shell hydrogenburning progenitors may evolve too slowly on their first trip to the blue in the HR diagram to be able to ionize the ejected common envelope material before it merges with the interstellar medium. However, following subsequent hydrogen shell flashes during which envelopes of mass $\sim 1-10 \times 10^{-4} \mathrm{M}_{\odot}$ are ejected through Roche-lobe overflow, the remnant travels to the blue rapidly enough to excite the emitted matter (Iben and Tutukov 1986a) and, if it is massive enough $\left(>0.4 \mathrm{M}_{\odot}\right)$, the system may become a detectable PN. But, because the lifetime of the central star is so short during its most luminous state and because the nebular material is still very close to the central star, the probabilty of seeing such a system is not very large. In mergers of low mass degenerate dwarfs a small common envelope may be ejected and lit up be the merged product. We note that at least one case of an extremely low mass $\left(\sim 10^{-8} \mathrm{M}_{\odot}\right)$ PN exists (Liebert, this volume).

One intriguing final possibility which has not yet been explored numerically is that, in a common envelope event initiated by the secondary, the cold CO white dwarf remnant of the primary can accrete enough material to reignite hydrogen and be thereby resurrected to become the dominant ionizing star for the nebula ejected by the secondary.

\section{OBSERVED PLANETARY NEBULAE WITH BINARY NUCLEI}

Approximately 10 planetary nebulae are known to contain a central star of established duplicity (Bond 1987, Ritter 1987) and the characteristics of several provide direct evidence for the common envelope ejection hypothesis (e.g., Bond 1976, Grauer and Bond 1983, Bond 1985, Drilling 1985, Bond and Grauer 1987, Bond and Grauer 1987).

We will attempt to reconstruct the prior evolution of four of these systems. We emphasize at the outset, however, that the results of this sort of exercise are not only sensitive to the theoretical assumptions, but are exceedingly sensitive to the stellar and orbital characteristics estimated from the observations. Because the theory of binary star evolution is in such a rudimentary state, it is crucial for its development that every effort be made to establish these observational characteristics as carefully as current technology will allow.

V651 Mon (the central star of NGC 2346) consists of a hot dwarf of mass $~ 0.4$ and an unevolved main sequence star of mass $\sim 1.8$ (Mendez and Niemala 1981). The orbital period is 16 days 
and so the current orbital separation is $A_{f}-38$. If a common envelope event has been responsible for the $\mathrm{PN}$, the mass of the initial primary must have been larger than 1.8 , and we infer that the amount of mass in the PN must be of the order of 1.5 , regardless of the details of the binary scenario we choose.

Suppose first that the dwarf component is a helium degenerate dwarf descended from a star initially less massive than 2.3. From equations (2), (3), and (6) we infer that $M_{1} \sim 2 \alpha^{1 / 2}$ or that $0.8<\alpha$ $<1.0$. Thus, in this scenario, $\alpha$ is constrained to be within very narrow limits. We illustrate the position of the progenitor system in Fig. 1. One worry with this scenario is that $A_{f}$ is so large that one cannot invoke the argument of excess stripping and contraction on a rapid time scale to counter the inference from computed tracks that the PN will be a lazy one.

As a second possibility, suppose that the hot dwarf is a hybrid object descended from a star of mass $~ 3$ (Iben and Tutukov 1985) which filled its Roche lobe in a system with $A_{0}$ between curves $C$ and $D$. We have from equation (2) that $A_{0} \sim 475 / \alpha$, but this violates the assumption that $A_{0}$ is smaller than given by curve $\mathrm{D}\left(\mathrm{M}_{1}-3\right)$. We can also dismiss a system consisting initially of two objects of about the same mass 1.1. Not only is a common envelope not formed (mass transfer is conservative and very slow), but the observed properties of Algols precludes the chosen initial conditions (Kraitcheva et al. 1986).

One final possibility remains to be considered. If the mass of the hot dwarf is in reality somewhat larger than 0.4 , the progenitor system could fall somewhere between curves $D$ and $E$ in Fig. 1, as indicated by the arrow in this figure. That is, the primary could have been an early AGB star of mass $\sim 2-3$ which filled its Roche lobe after developing a CO core of mass 0.50.55 (Iben 1986). The value of $A_{0}$ derived from equation (2) is quite consistent with this interpretation. A particular virtue of this scenario is that the resulting PN will not be a lazy one. It would be nice if more precise limits on the mass of the hot dwarf could be set.

LSS 2018 consists of a hot CO dwarf of mass 0.55 and a main sequence star of mass 0.25 at a separation of about 2.2 (Ritter 1987). With $\mathrm{A}_{0} \sim 16 \mathrm{M}_{1}{ }^{2} / \alpha$, a $\mathrm{CO}$ dwarf is derivable from a primary of mass in the range 2.3-4.0 and we see from Fig. 1 that this is a quite standard example of a binary forming a PN. The predicted mass of the ejected common envelope is also in this case quite high, $1 M_{\odot}$, and observational estimates would be helpful in deciding on the reasonableness of this estimate.

MT Ser (the central star of Abell 41) has the remarkably short orbital period of $2^{\text {h }} 43^{\mathrm{m}}$ (Grauer and Bond 1983, Bond and Grauer 1987). There are no known cataclysmic binaries with a similar period. The mass of the secondary as deduced from its radius is $\sim 0.2$ and the effective temperature of the primary is $\sim 60000 \mathrm{~K}$. If the mass of the primary is $\sim 0.6, \mathrm{~A}_{\mathrm{f}} \sim 1$ and, from equation (2), $A_{0} \sim 8 M_{1}{ }^{2} \alpha^{-1}$, placing the progenitor system near LSS 2018 in Fig. 1 if $M_{1} \sim 2.3-4$. 
UU Sge consists of a hot dwarf of mass $\sim 0.9-1.1$ and a main sequence star of mass 0.6-0.7 with $A-3-3.3$ (Bond, Liller, and Mannery 1978, Ritter 1987). From equation (2), we have $A_{0} \sim 5$ $\mathrm{M}_{1}^{2} / \alpha$. For $\alpha>0.5$, this implies that the primary filled its Roche lobe with $A_{0}$ near curves $C$ and $D$ in Fig. 1. Bond (1976) first noticed the coincidence in position between the planetary nebula Abell 63 (Abell 1966) and UU Sge, whose variability had been established by Hoffleit (1932). Tsessevich (1977), Miller, Krzeminski, and Priedhorsky (1976), and Bond, Liller, and Mannery (1978) established that UU Sge is an eclipsing binary with a period of $11.2 \mathrm{hr}$. From the light curve, which shows both a primary and a secondary eclipse, Bond et al infer, for an inclination angle $\iota \sim 85^{\circ}$, that $R_{1} / A=0.13$ and $R_{2} / A=0.24$. Assuming that the secondary is a $\mathrm{dK}$ main sequence star of mass $M_{2}=0.7$ and of radius $R_{2} \sim 0.7$, they estimate $M_{1} \sim 0.9$, giving $R_{1} \sim 0.4$ and $A \sim 3$. Finally, they estimate a distance of $1 \mathrm{kpc}$ for the system and a luminosity of $L_{1} \sim\left(10^{1.9}-10^{2.1}\right)$ for the primary.

The primary is an sdo star (Miller, Krzeminski, and Priedhorsky 1976) and its luminosity, radius, and surface temperature as estimated by Bond et al. are reminiscent of the properties of theoretical helium stars that are predicted to form in common envelope events. From Iben and Tutukov (1985) we have that, during the major core helium-burning phase following emergence from the common envelope phase,

$$
\begin{aligned}
& \mathrm{L}_{\mathrm{He}}-10^{2.37} \mathrm{M}_{\mathrm{He}}{ }^{3.98}, \mathrm{R}_{\mathrm{He}}-10^{-0.41} \mathrm{M}_{\mathrm{He}}{ }^{1.18}, \\
& \mathrm{~T}_{\mathrm{He}}-36000 \mathrm{~K} \mathrm{M}_{\mathrm{He}} 0.4,
\end{aligned}
$$

where $\mathrm{M}_{\mathrm{He}}$, $\mathrm{L}_{\mathrm{He}}$, and $\mathrm{R}_{\mathrm{He}}$ are the mass, luminosity, and radius of the helium star in solar units, and $\mathrm{T}_{\mathrm{He}}$ is the surface temperature. Setting $\mathrm{L}_{\mathrm{Be}}=\left(10^{1.9}-10^{2} \cdot 1\right)$ in equation (7) gives $\mathrm{M}_{\mathrm{He}}$ 0.76-0.86. From Iben and Tutukov (1985), the mass of the progenitor of the helium star would have been in the range $M_{1}$ $(5-7)$ and, from $P=365^{d}\left(A_{f} / 216\right)^{3 / 2} / M_{t \circ t} 1 / 2$, we have $A_{f} \sim 2.89$ 2.95, which then, using the Bond et al. R/A ratios, implies $R_{2}$ 0.7 and $R_{s d o} \sim 0.38$. Equation (7) gives $R_{\mathrm{He}} \sim 0.28-0.33 \sim$ $0.75 \mathrm{R}_{\mathrm{sdO}}$ and $\mathrm{T}_{\mathrm{He}}-(32000 \rightarrow 36000) \mathrm{K}$, which is large enough to understand the ionization of some portion of the ejected nebula. Due both to the roughness of the stellar models (including the uncertainty in the mass of the hydrogen-rich surface layer) which provide the estimate of the radius of a helium star and to the uncertainty in the observational estimate of radius, the discrepancy between $R_{H e}$ and $R_{s d o}$ is not a serious concern.

The ease with which we can explain many of the features of UU Sge in terms of a core helium-burning star is deceptive. We note that the size of the sdo star is substantially smaller than the radius of the Roche lobe about this star, $R_{L} \sim 1.2$. If the stellar remnant which emerges from the common envelope event retains a sufficiently massive hydrogen envelope, it will evolve 
on a long time scale burning hydrogen in a shell at luminosities larger than and at surface temperatures smaller than given by equations (7) before it ignites helium at its center. From Fig. 1 of Iben and Tutukov (1985) we see that the rate at which the radius of a model of an $0.76 \mathrm{M}_{0}$ shell hydrogen-burning star changes with time as it approaches the helium-burning main sequence is of the order of $\mathrm{d} \log \mathrm{R} / \mathrm{dt} \sim 0.3 / 10^{6}$ yr when $\mathrm{R} \sim \mathrm{R}_{\odot}$. The implication is that the sdo star in UU Sge may have required of the order of $2 \times 10^{6} \mathrm{yr}$ to reach its present state after detaching from its Roche lobe. It may therefore not yet have ignited helium at its center and so our estimates based on equations (7) might be wholly inappropriate.

However, the lifetime of a typical planetary nebula is orders of magnitude less than $10^{6} \mathrm{yr}$, which means that the sdo star cannot have required as much time as suggested by the formal quasistatic models whose evolution rate is controlled by the rate of hydrogen burning in a shell. What may have happened in the real situation is that, when Roche-lobe detachment occurred, the radius of the mass-losing precursor of the sdo star was considerably larger than the radius of a constant-mass model in quasistatic equilibrium and that the mass of hydrogen-rich matter remaining near the surface was much less than that necessary to sustain hydrogen-burning in quasistatic equilibrium at high luminosity. The fact that the spectrum of the ionizing star is sdo rather than sdB supports this interpretation. Immediately after Roche-lobe detachment, the surface layers of the precursor of the sdo star, inflated by dynamic effects, may have begun to contract on a time scale less than the thermal one. The surface layers of the sdo star may now be contracting on a thermal time scale. Helium has probably ignited at the center, thus accounting for the current luminosity.

The current masses and separation of the components of UU Sge permit us to estimate an appropriate value for the parameter $\alpha$. From Iben and Tutukov (1985), $M_{1 R}=0.76$ implies $M_{1} \sim 5$. Setting $M_{2}=0.7$ and $A_{f}=3$ in equation (2) gives $A_{0}=141 / \alpha$. But, in order for the remnant to become a helium star, we have that the radius of the Roche lobe of the primary before it fills its Roche lobe must be less than $R_{*} \sim 10^{0.24} \mathrm{M}_{1}{ }^{2.25}$ (used to establish curve $D$ in Fig. 1). This means that $R_{1 L} \sim 0.49 A_{0}=69 / \alpha<65$ or that

$\alpha>1$ ! Had we selected $M_{1 R}=0.86$, then $M_{1}=5.5$ and $\alpha>0.86$. Since curve $D$ is model and composition dependent, we must not take these estimates too literally. We may, however regard them as confirmation of our anticipation that, for initially widely spaced components of quite unequal masses, the parameter $\alpha$ is indeed of the order of unity.

As a final word of caution, however, we note that our placement of UU Sge progenitor parameters in Fig. 1, is very close to the critical curves $C$ and $D$, and that any change in the parameters of the current system inferred from the observations could alter the most likely scenario considerably. This strengthens the lesson of V651 Mon. 


\section{COMPARISONS BETWEEN PNe OF SINGLE AND OF BINARY ORIGIN}

The nature of the (super)wind that produces most PNe is still unclear. It is possible that pulsational instability of a red supergiant envelope in combination with radiation pressure on dust and molecules can produce the mass-loss rate required by the observations, but an explicit demonstration from first principles is still missing. In contrast, it is clear that common envelope action in close binaries can eject large quantities of matter on a very short time scale.

In addition, binary scenarios provide a natural way of accounting for the bipolar structure and multiple shells seen in many PNe (Balick 1987, Chu and Jakoby 1987, Chu, this volume). There are at least two ways in which a binary core can influence nebular shape. In close binaries, angular momentum from the stellar orbit can be transferred in an axially symmetric way to the material ejected in a common envelope event. In wide binaries, if the orbital velocity of the components exceeds a typical nebular expansion velocity $\left(-10 \mathrm{kms}^{-1}\right)$, the motion of the superwind material ejected by one of the components can be influenced by the second component. Thus, all binaries with $A_{0}<10^{4}$ are possible precursors of PNe with cylindrical symmetry. Consequently (see equation [1]), perhaps half of all PNe have experienced shaping by a central binary. Of course, the wind emitted by the ionizing star will also exert a shaping influence (Kwok, Purton, and FitzGerald 1978, Kwok 1982, 1987, Kahn 1982) and, under the proper conditions, can also produce bipolar and shell effects which might be difficult to disentangle from those due to duplicity.

One of the most important pieces of information required for a further development of our understanding of close binary evolution is a firm, empirically based estimate of the value of the parameter $\alpha$ describing the degree of orbital shrinkage during the common envelope stage. The example of V651 Mon shows that fairly precise estimates can be made in those situations where the ionizing star has a degenerate helium core. This is because, between the mass of such a core and the radius of the precursor red giant, there exists a tight relationship which can be used to estimate the orbital separation of the precursor system. However, a definitive estimate by this means requires that the mass of the ionizing star can be unambiguously determined to be less than $\sim 0.45$.

PNe formed around close binaries can have systematiclly different chemistrys which could help to distinguish the two. Since the evolution of close binary components does not proceed as far as the evolution of single stars, the chemical composition of the nebulae around close binaries might be expected to be less chemically evolved than the nebulae around single stars. Certain$1 y$, because the range in $A_{0}$ which will allow a primary component to reach the TP-AGB phase and begin to dredge up carbon and $s$-process isotopes ( $A_{0}$ between curves $E$ and $F$ in Fig. 1 ) is so 
narrow relative to the full range of possible separations that one does not expect to find many $\mathrm{PNe}$ with close double cores which exhibit overabundances of these elements. In contrast, single stars which produce PNe do so after becoming TP-AGB stars and developing chemically enriched surface compositions. Thus, most $\mathrm{PNe}$ with $\mathrm{C} / \mathrm{O}>1$ are the result of single star evolution.

On the other hand, the range in $A_{0}$ which allows the primary to fill its Roche lobe after it has exhausted central helium, but before it has begun to thermally pulse, is much larger ( $A_{0}$ between curves $D$ and $E$ in Fig. 1). Further, if its mass is larger than $M 1$ - 4-5, the primary will experience a second dredge-up episode and develop overabundances of ${ }^{14} \mathrm{~N}$ and ${ }^{4} \mathrm{He}$ (Kaler, Iben, and Becker 1978), Becker and Iben 1980). Hence, some PNe with close double cores should exhibit overabundances of these two elements. Final$1 y$, PNe which originate from systems in which either component has filled its Roche lobe for the second time should be essentially pure ${ }^{4} \mathrm{He}$ and ${ }^{14} \mathrm{~N}$. Finding such a $\mathrm{PN}$ would be the most convincing evidence of all for the reasonableness of binary scenarios for PN formation.

It is a pleasure to thank You Hua Chu and James Kaler for helpful comments on the manuscript.

\section{REFERENCES}

Abe11, G. 1966, Ap. J., 144, 259.

Balick, B. 1987, in Late Stages of Stellar Evolution, ed. S. Kwok and S. R. Pottasch (Dordrect: Reide1), p. 413.

Becker, S. A., and Iben, I. Jr. 1980, Ap. J., 237, 111. Bodenheimer, P., and Taam, R. E. 1984, Ap. J., 287, 771.

Bond, H. E. 1987, this volume.

Bond, H. E. 1976, Pub. Astr. Soc. Pac., 88, 192. - 1985, in Cataclysmic Variables and Low-Mass X-ray Binaries, ed. D. Q. Lamb and J. Patterson (Reidel: Dordrecht), p. 15.

Bond, H. E., and Grauer, A. D. 1987, in Faint Blue Stars, ed. L. Davis Philip (Schenectady: L. Davis), in press.

Bond, H. E., and Livio, M. 1987, in preparation.

Bond, H. E., Liller, W., and Mannery, E. J. 1978, Ap. J., 223, 252 .

Chu, Y.-U. 1987, this volume.

Chu, Y.-U., and Jakoby, G. 1987, in Late Stages of Stellar Evolution, ed. S. Kwok and S. R. Pottasch (Dordrecht: Reidel), p. 351.

Draine, B. T. 1981, in Physical Processes in Red Giants, ed. I. Iben, Jr. and A. Renzini (Dordrecht: Reide1), p.317.

Drilling, J. S. 1985, Ap. J. Lett., 294, L107.

Fadeev, Y. 1986, in Heidelberg I.A.U. Symposium.

Hachisu, I. 1987, this volume.

Grauer, A. D., and Bond, H. E. 1983, Ap. J., 271, 259. . 1984, Ap. J., 277, 211.

Hoffleit, D. 1932, Harvard Bull., No. 887. 
Iben, I. Jr. 1986, Ap. J., 304, 217.

- 1987, in Late Stages of Stellar Evolution, ed. S. Kwok and

S. R. Pottasch (Dordrecht: Reide1), p. 175.

Iben, I. Jr., Fujimoto, M. Y., Sugimoto, D., and Miyaji, S. 1986, Ap. J., 304, 217.

Iben, I. Jr., and Tutukov, A. V. 1984a, Ap. J. Suppl., 54, 335.

. 1984b, Ap. J., 284, 719 .

. 1985, Ap. J. Suppl., 58, 661.

- $1986 \mathrm{a}, \mathrm{Ap} . \mathrm{J} ., 311,742$.

. $1986 \mathrm{~b}, \mathrm{Ap} . \mathrm{J} ., 311,753$.

. 1987, Ap. J., 313, 727.

Kahn, F. D. 1982, in Planetary Nebulae, ed. D. R. Flower (Dordrecht: Reide1), p. 305.

Kaler, J. B., Iben, I. Jr., and Becker, S. A. 1978, Ap. J. Lett., $224, \mathrm{~L} 63$.

Kraicheva, Z. T., Tutukov, A. V., and Yungelson, L. R. 1986, Astrofizica, 24, 287.

Kwok, S. 1982, Ap. J., 258, 280.

. 1987, in Late Stages of Stellar Evolution, ed. S. Kwok and

S. R. Pottasch (Dordrecht: Reide1), p. 321.

Kwok, S., and Pottasch, S. R., eds., 1987, Late Stages of Stellar Evolution (Dordrecht: Reidel).

Kwok, S., Purton, G. R., and FitzGerald, M. P. 1978, Ap. J. Lett., $219, \mathrm{~L} 125$.

Lauterborn, D. 1970, Astr. Ap., 7, 150.

Mendez, R. H. , and Niemola, V. S. 1981, Ap. J., 250, 240.

Meyer, F. and Meyer-Hofmeister, E. 1979, Astron. Ap., 78, 167.

Miller, J. S., Krzeminski, W., and Priedhorsky, W. 1976, I.A.U. Circ., No. 2974.

Paczynski, B. 1976, in IAU Symposium No. 73, Structure and Evolution of Close Binary Systems, ed. P. Eggleton, S. Mitton, and J. Whelan (Dordrecht: Reide1), p. 75.

Popova, E. I., Tutukov, A. V., and Yungelson, L. R., 1982, Ap. Space Sci., 88, 155.

Renzini, A. 1979, in Stars and Star Systems, ed. B. E. Westerlund (Dordrecht: Reidel), p. 155.

. 1983, in Planetary Nebulae, ed. D. R. Flower (Dordrecht: Reide1), p. 267.

Ritter, H. 1987, in Catalogue of CV's, LMXB's, and Related Objects, Max Planck preprint 285.

Tessevich, V. P. 1977, I.A.U. Inf. Bull. Var. Stars, No. 1320.

Tuchman, Y., Sack, N., and Barkat, Z. 1979, Ap. J., 234, 217.

Tutukov, A. V. 1980, in Fundamental Problems in the Theory of Stellar Evolution, ed. D. Sugimoto, D. Q. Lamb, and D. N. Schramm (Dordrecht: Reide1), p.37.

- 1985, in Cataclysmic Variables and Low-Mass X-ray Binaries, ed. D. Q. Lamb and J. Patterson (Reidel: Dordrecht), p.xxx

Tutukov, A. V., Fedorova, A. B., and Yungelson, L. R. 1982, Pis'ma Astron. $\mathrm{Zh}, 8,365$.

Tutukov, A. V., and Yungelson, L. R. 1979, Acta. Astron., 29, 665. 
Tutukov, A. V., Fedorova, A. B., and Yungelson, L. R. 1982, Pis'ma Astron. Zh., 8, 365 .

Verbunt, F., and Zwaan, C. 1981, Astron. Ap., 100, L7.

Willson, L. A. 1987, in Late Stages of Stellar Evolution, ed. S. Kwok and S. R. Pottasch (Dordrecht: Reidel), p. 197.

Wood, P. R. 1974, Ap. J., 190, 609. . 1981, in The Physics of Red Giant Stars, ed. I. Iben, Jr., and A. Renzini (Dordrecht: Reidel), p. 135. 